Supplementary information:

\title{
Direct and Facile Room-Temperature Synthesis of Nanocrystalline Calcium Sulfate Dihydrate (Gypsum)
}

\author{
Kapil Gupta, Shubra Singh, and M. S. Ramachandra Rao
}

Table S1: Refined parameters and agreement factors obtained from Rietveld refinement of the powder x-ray diffraction data of gypsum $\left(\mathrm{CaSO}_{4} \cdot 2 \mathrm{H}_{2} \mathrm{O}\right)$ with space group $C 2 / c$. Estimated errors in the last digits are given in parentheses.

\begin{tabular}{|c|c|c|c|c|c|c|}
\hline \multicolumn{2}{|c|}{ Space Group } & \multicolumn{2}{|c|}{ Lattice parameters } & \multicolumn{2}{|c|}{ R-factors } & \\
\hline \multicolumn{2}{|c|}{ C 1 2/c 1 (\#15) } & \multicolumn{2}{|c|}{$\begin{aligned} a & =6.285 \AA \\
b & =15.210 \AA \\
c & =5.677 \AA \\
\beta & =114.09^{\circ} \\
V & =495.45 \AA^{3}\end{aligned}$} & \multicolumn{2}{|c|}{$\begin{array}{c}\mathrm{R}_{\mathrm{p}}=14.3 \% \\
\mathrm{R}_{\mathrm{wp}}=12.5 \% \\
\mathrm{R}_{\text {exp }}=6.03 \% \\
\mathrm{R}_{\text {Bragg }}=7.33 \% \\
\mathrm{R}_{\mathrm{f}}=5.40 \% \\
\end{array}$} & $\begin{array}{c}\chi^{2}=4.30 \\
\text { GoF-Index }= \\
2.1\end{array}$ \\
\hline Atom & $\mathbf{x}$ & $\mathbf{y}$ & $\mathbf{z}$ & & Occ. & \\
\hline $\mathbf{C a}$ & 0 & 0.1697 & 0.25 & & 0.4044 & \\
\hline $\mathbf{S}$ & 0 & 0.3273 & 0.75 & & 0.4062 & \\
\hline 01 & 0.7860 & 0.0676 & 0.9128 & & 1.2015 & \\
\hline $\mathbf{O 2}$ & 0.2104 & 0.3808 & 0.9248 & & 0.8935 & \\
\hline $\mathbf{O 3}$ & 0.0844 & 0.2730 & 0.5880 & & 0.8045 & \\
\hline Atom & $\beta_{11}\left(\AA^{2}\right)$ & $\overline{\beta_{22}}\left(\AA^{2}\right)$ & $\overline{\beta_{33}\left(\AA^{2}\right)}$ & $\beta_{12}\left(\AA^{2}\right)$ & $\beta_{13}\left(\AA^{2}\right)$ & $\boldsymbol{\beta}_{23}\left(\AA^{2}\right)$ \\
\hline $\mathbf{C a}$ & 0.0041 & -0.0004 & 0.0006 & 0 & 0.0030 & 0 \\
\hline $\mathbf{S}$ & 0.0095 & 0.0007 & 0.0081 & 0 & 0.005 & 0 \\
\hline 01 & 0.0615 & 0.0045 & 0.0525 & 0.0015 & 0.0099 & -0.0031 \\
\hline $\mathbf{O 2}$ & 0.0153 & 0.0011 & 0.0214 & -0.0029 & 0.0061 & 0.0073 \\
\hline $\mathbf{O 3}$ & -0.0043 & 0.0017 & 0.0018 & -0.0029 & 0.0070 & -0.0011 \\
\hline
\end{tabular}


The crystallographic structure factor $F_{k}$ was calculated in FullProf by using the formula:

$$
\begin{aligned}
F_{k}=\sum_{j=1}^{n} N_{j} & f_{j} \exp \left[2 \pi i\left(h x_{j}+k y_{j}+l z_{i}\right)\right] \\
& \quad \times \exp \left[-\left(\beta_{11} h^{2}+\beta_{22} k^{2}+\beta_{33} l^{2}+2 \beta_{12} h k+2 \beta_{13} h l+2 \beta_{23} k l\right)\right]
\end{aligned}
$$

Here, $\beta_{11}, \beta_{22}, \beta_{33}, \beta_{12}, \beta_{13}, \beta_{23}$ are anisotropic temperature parameters, and $f_{j}$ is the scattering length of the atom $j$. 


\title{
Direct and Facile Room-Temperature Synthesis of Nanocrystalline Calcium Sulfate Dihydrate (Gypsum)
}

\author{
Kapil Gupta, Shubra Singh, and M.S. Ramachandra Rao
}

The interesting morphological changes observed during e-beam irradiation (above discussion) motivated us to study e-beam induced phase transformations, if any. Hence, Figure S1 shows the electron beam-induced phase transformation of single-crystalline gypsum to polycrystalline $\mathrm{CaO}$ nanoparticles.
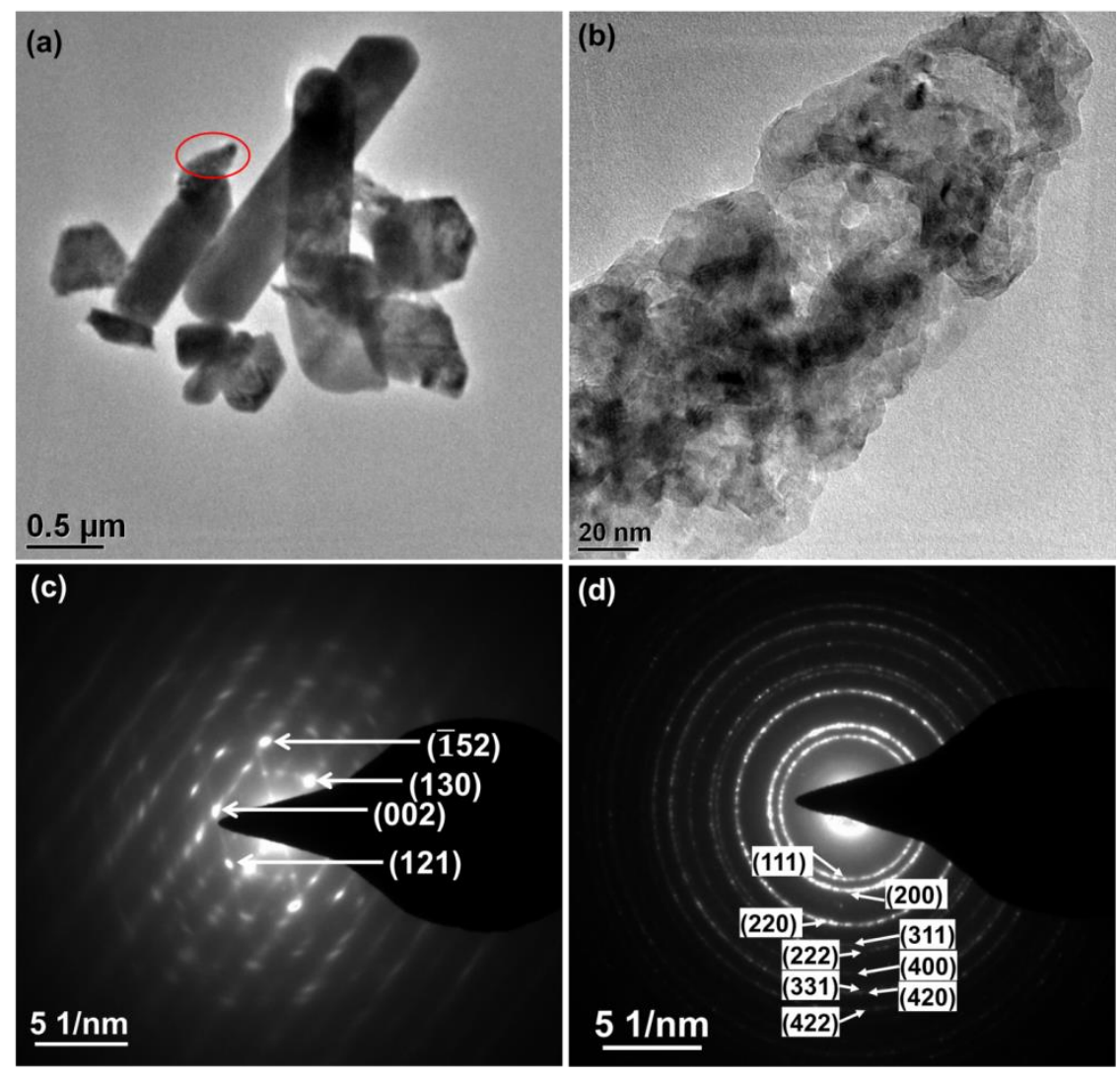

Figure S1: Electron beam irradiation effect on as-obtained gypsum. (a) Bright-field TEM image of gypsum particles, (b) TEM of a part of gypsum particle (marked by red oval in (a)) after irradiating with $\mathrm{e}^{-}$beam to transform the whole gypsum particle into $\mathrm{CaO}$ nanoparticles, (c) SAED of marked part in (a) showing the single-crystalline pattern of gypsum, and (d) SAED of (b) showing the presence of only polycrystalline rings corresponding to $\mathrm{CaO}$ nanoparticles. 
As discussed above, e-beam irradiation on a marked (with red oval) part of TEM image (Fig. $\mathrm{S} 1 \mathrm{a})$ converts the gypsum particle to $\mathrm{CaO}$ nanoparticles (Fig. S1b). A clear change in electron diffraction patterns before and after $\mathrm{e}^{-}$-beam irradiation can be seen from the comparison of Fig. S1c,d, where Fig. S1c shows the single-crystalline diffraction pattern (spot pattern) of gypsum particle and Fig. S1d shows the sharp polycrystalline ring pattern corresponding to $\mathrm{CaO}$ nanoparticles.

When gypsum is heated, it first converts in to $\mathrm{CaSO}_{4}$ (around $200{ }^{\circ} \mathrm{C}$ ), and then at very high temperatures (about $800-1000{ }^{\circ} \mathrm{C}$ ), it decomposes in to $\mathrm{CaO}$. During e-beam irradiation, the same phase transformation occurs, but not due to the heating effect, as the temperature rise inside TEM cannot be of this magnitude. During e-beam irradiation, elastic two-body collision effect of electrons and atoms is believed to activate such phase transitions [1]. Similar phenomenon has been observed in Calcite $\left(\mathrm{CaCO}_{3}\right)$, which also transform in to $\mathrm{CaO}$ upon in-situ e-beam irradiation [2]

\section{References}

[1] X. Sun et al., Small (2014) 10, 4711-4717

[2] U. Golla-Schindler et al., Microsc. Microanal. (2014) 20, 715-722 
Supplementary information:

\section{Direct and Facile Room-Temperature Synthesis of Nanocrystalline Calcium Sulfate Dihydrate (Gypsum)}

Kapil Gupta, Shubra Singh, and M.S. Ramachandra Rao

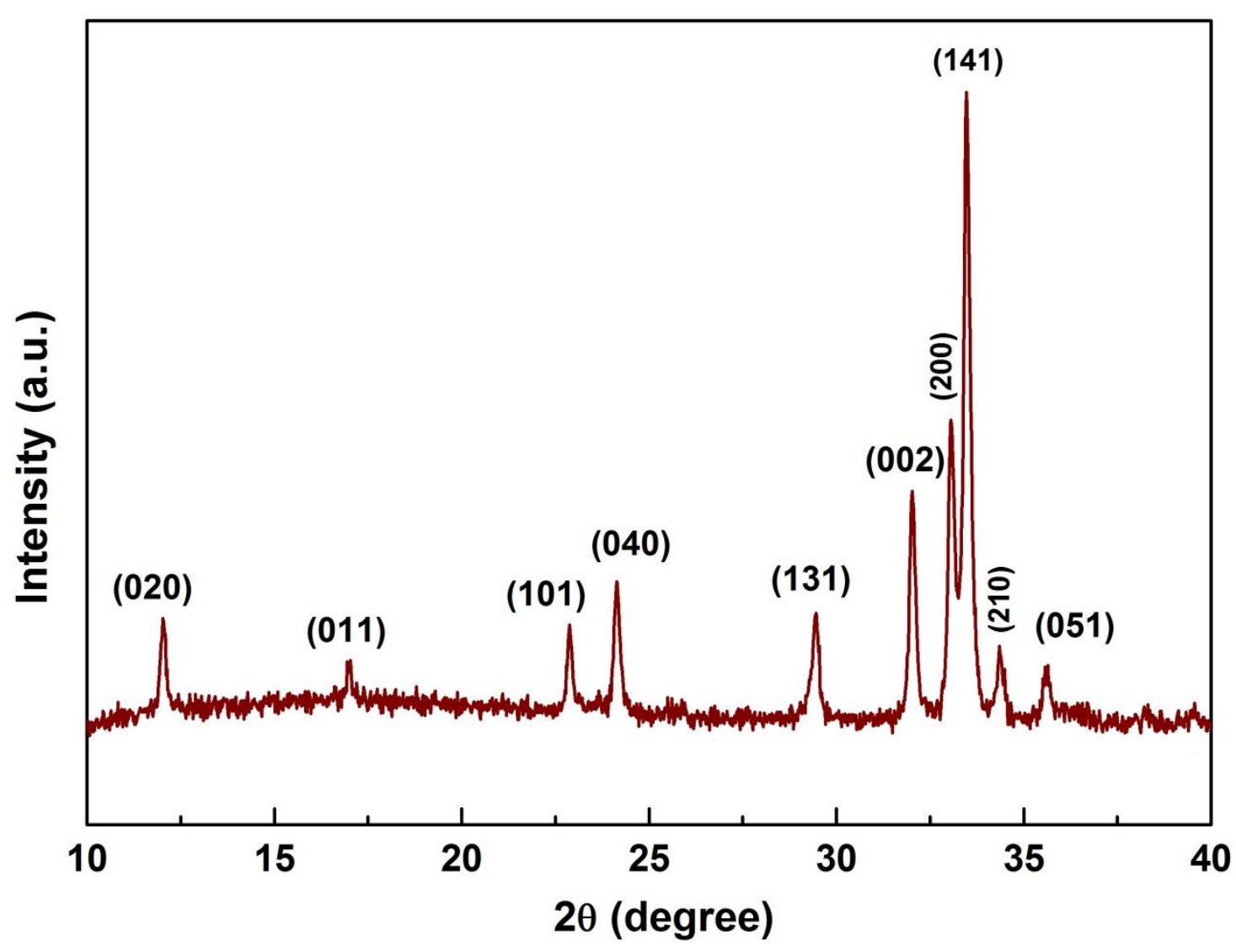

Figure S2: X-ray powder diffraction pattern of Brownmillerite nano- $\mathrm{CaFeO}_{2.5}$. 
Supplementary information:

\section{Direct and Facile Room-Temperature Synthesis of Nanocrystalline Calcium Sulfate Dihydrate (Gypsum)}

Kapil Gupta, Shubra Singh, and M.S. Ramachandra Rao

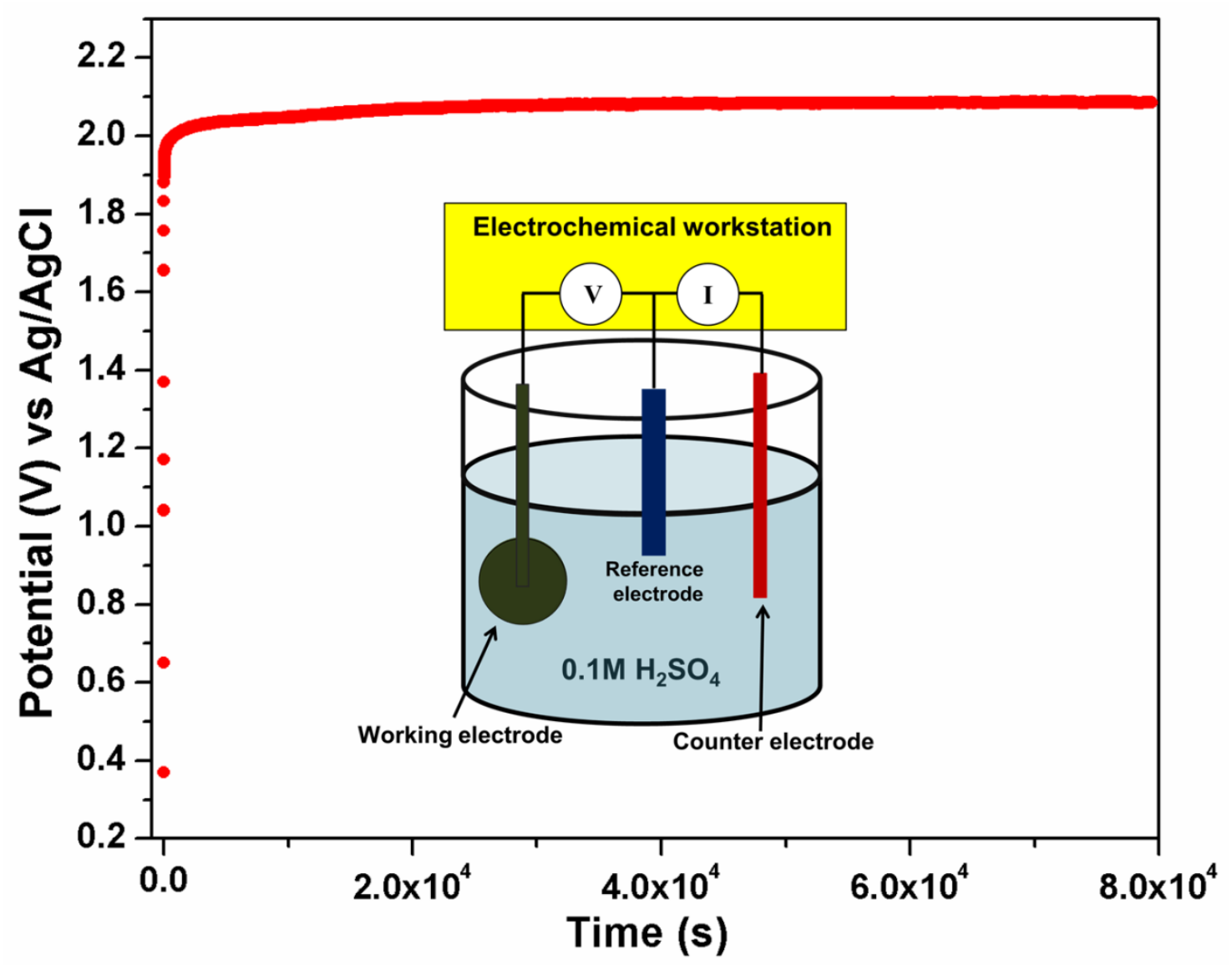

Figure S3: Electrochemical set-up and typical time dependence of the electrode potential. Electrochemical galvanostatic oxidation of Brownmillerite nano- $\mathrm{CaFeO}_{2.5}$ was performed at room temperature by means of $\mathrm{CHI660D}$ electrochemical workstation in the acidic medium of $0.1 \mathrm{M} \mathrm{H}_{2} \mathrm{SO}_{4}$ with the anodic current of $0.5 \mathrm{~mA}$. The pellet of $\mathrm{CaFeO}_{2.5}$ with the use of platinum wire $(0.1 \mathrm{~mm}$ dia $)$ attached to the periphery of the pellet was used as a working electrode for electrochemical oxidation, $\mathrm{Ag} / \mathrm{AgCl}$ reference electrode, and $\mathrm{Pt}$ wire was used as counter electrode. Time dependence of electrode potential of nano- $\mathrm{CaFeO}_{2.5}$ is shown. 
Supplementary information:

\section{Direct and Facile Room-Temperature Synthesis of Nanocrystalline Calcium Sulfate Dihydrate (Gypsum)}

Kapil Gupta, Shubra Singh, and M.S. Ramachandra Rao

TGA study on the mixtures with $0 \mathrm{wt} \%$ and $5 \mathrm{wt} \%$ nano-gypsum contents are shown below:

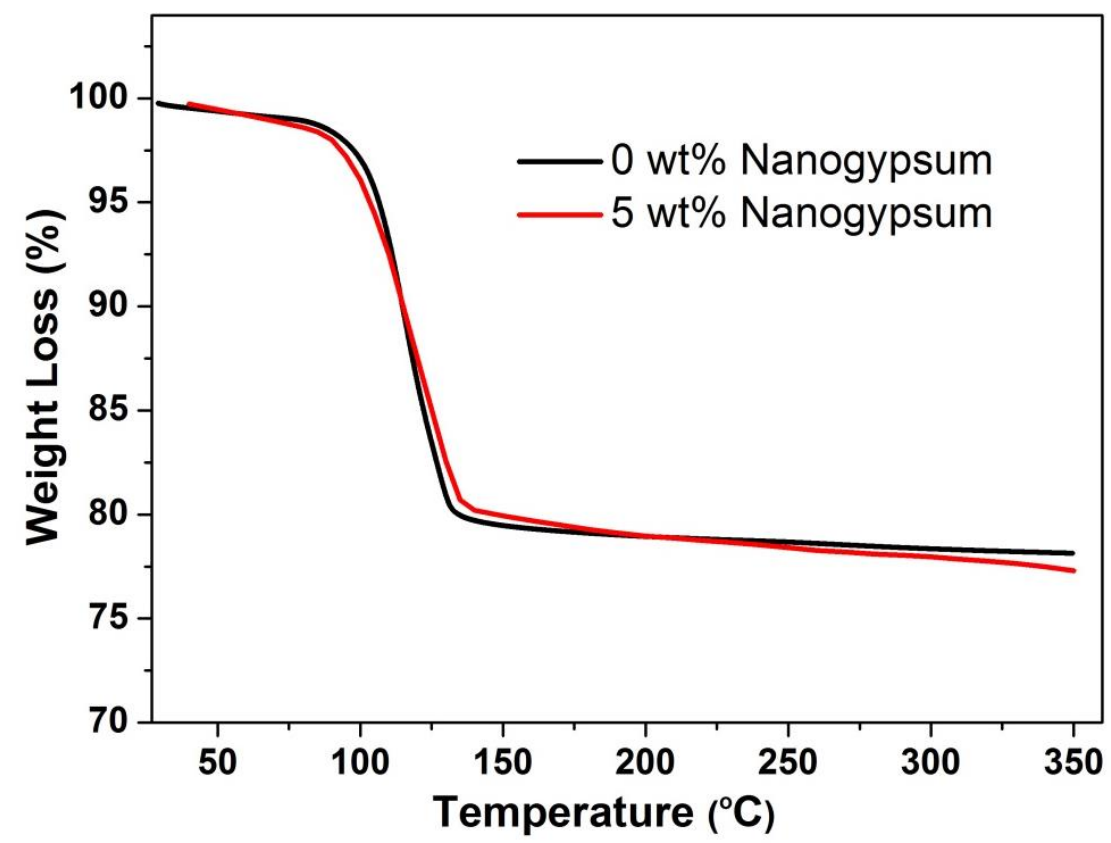

Figure S4: Thermogravimetry analysis of gypsum mixtures (with 0wt \% and 5 wt $\%$ nanogypsum added to commercial gypsum). 
Supplementary information:

\section{Direct and Facile Room-Temperature Synthesis of Nanocrystalline Calcium Sulfate Dihydrate (Gypsum)}

Kapil Gupta, Shubra Singh, and M.S. Ramachandra Rao
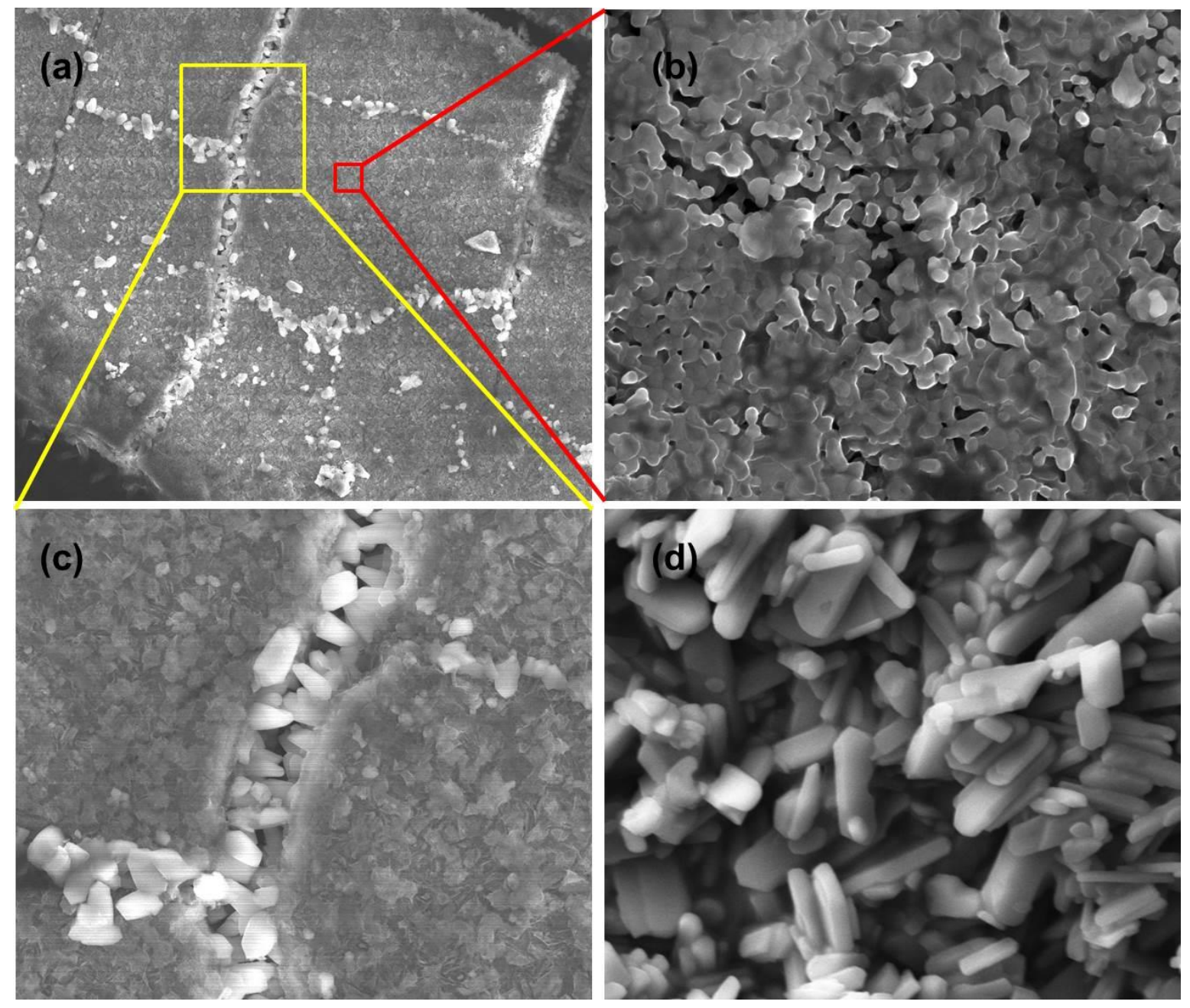

Figure S5: (a) FESEM of Brownmillerite nano- $\mathrm{CaFeO}_{2.5}$ pellet at an intermediate step of electrochemical oxidation in $0.1 \mathrm{M} \mathrm{H}_{2} \mathrm{SO}_{4}$. (b) Magnified image of unreacted $\mathrm{CaFeO}_{2.5}$ phase. (c) Formation of gypsum can be seen within the micro-cracks. (d) Morphology of the as-formed nano-gypsum after complete electrochemical oxidation of Brownmillerite nano- $\mathrm{CaFeO}_{2.5}$. (original SEM images of Figure 3) 